\title{
Comparação do sinal EMG e das características da passada em diferentes protocolos de corrida incremental
}

CDD. 20.ed. 616.7407547

796.42092

\author{
Carina Helena Wasem FRAGA* \\ Roberto BIANCO* \\ Mauro GONÇALVES ${ }^{* *}$
}

*Escola de Educação Física e Esporte, Universidade de São Paulo.

${ }^{* *}$ Departamento de Educação Física, Universidade Estadual

Paulista - Rio Claro.

\section{Resumo}

0 objetivo do presente estudo foi comparar o sinal eletromiográfico, a frequência e a amplitude de passada entre diferentes intensidades de corrida: 60\%, 80\% e 100\% da velocidade máxima em dois protocolos incrementais. Participaram deste estudo 11 corredores do sexo masculino. Os protocolos de corrida foram realizados com velocidades iniciais de $10 \mathrm{~km} \cdot \mathrm{h}^{-1}$, com incrementos de $1 \mathrm{~km} \cdot \mathrm{h}^{-1}$ a cada três minutos até a exaustão, que diferiram em relação ao intervalo entre cada estágio incremental: 30 e 120 segundos. Foram analisados valores RMS dos músculos iliocostal lombar, reto femoral, vasto lateral, vasto medial, bíceps femoral, tibial anterior, e gastrocnêmio, e a amplitude e frequência de passada. Os valores RMS mostraram aumento entre as intensidades para quase todos os músculos, e não foram influenciados pelo tipo de protocolo utilizado para maioria dos músculos. A frequência e amplitude de passada apresentaram contribuições percentuais diferenciadas para o aumento da velocidade de corrida.

UNITERMOS: Eletromiografia; Cinemática; Corredores.

\section{Introdução}

A corrida é um dos mais comuns tipos de locomoção humana e estudos têm adotado a eletromiografia de superfície como técnica não-invasiva capaz de avaliar ativação muscular durante esse movimento específico (Abe, Muraki, Yanagawa, Fukuoka \& Nithata, 2007; Smoliga, Myers, RedFern \& Lephart, 2010). Para análise do sinal eletromiográfico (EMG) durante a corrida, frequentemente, são empregados protocolos incrementais de corrida como instrumento de avaliação (Fraga, Silva \& GonÇalves, 2009; Hanon, Thépaut-Mathieu \& Vandewalle, 2005). Corridas com incremento de intensidade tendem a modificar a amplitude do sinal EMG, além de alterar as características da passada, como a Amplitude de Passada (AP) e a Frequência de Passada (FP). Essas alteraçōes nas referidas variáveis biomecânicas a partir de um teste incremental de corrida podem estar associadas tanto ao incremento da intensidade do exercício quanto ao início da fadiga neuromuscular (HANON, ThÉPAUT-MATHIEU, Hausswirth \& Le CheValier, 1998).

Embora exista uma tendência de aumento da amplitude do sinal EMG em resposta ao incremento da velocidade de corrida, possivelmente relacionado ao recrutamento adicional de novas unidades motoras (Basmajian \& DeLuca, 1985), estudos têm mostrado que o sinal EMG não apresenta um comportamento característico a partir da realização de um protocolo incremental de corrida (AvOGADRO, Dolenec \& Belli, 2003; Gazendam \& HoF, 2007; Karamanidis, ARAmpatzis \& BrÜgGemann, 2004).

Com relação aos parâmetros cinemáticos, sabe-se que corridas realizadas em altas intensidades podem acarretar em modificaçōes no comportamento da passada, (Avogadro, Dolenec \& Belli, 2003; Hanon, ThépautMathieu \& Vandewalle, 2005; Nummela, RusKo \& MERO, 1994), ou seja, alteraçōes nas variáveis AP e FP, as quais determinam diretamente a velocidade de locomoção (CAVANAGH \& Kram, 1989; Martin \& SANDERSON, 2000). Nesse sentido, embora a corrida possa ser classificada como uma atividade de deslocamento natural do ser humano, aspectos como a escolha adequada da AP e da FP podem influenciar o seu desempenho (FraGA, BloEdow, Guimaräes \& VAZ, 2007), principalmente considerando situações de alta intensidade. 
Para análise de diferentes intensidades de corrida, estudos sugerem a classificação deste exercício nos domínios moderado, pesado e severo. Essa classificação tem como base as variações em parâmetros metabólicos em testes incrementais, tais como, lactato sanguíneo e consumo de oxigênio (CAMIC, Housh, JoHnson, HeNdRIX, Zuniga, MielKe \& SCHMidT, 2010; CARTER, Jones \& Doust, 2000; Carter, Pringle, Jones \& Doust, 2002), que podem ser relacionadas a intensidades relativas à velocidade máxima de corrida (Vmáx.) correspondentes a, aproximadamente, $60 \%$ da Vmáx (domínio moderado), 80\% da Vmáx (domínio pesado) e $100 \%$ da Vmáx (domínio severo). O domínio moderado inclui intensidades de exercícios nas quais há um equilíbrio entre a produção e a remoção de lactato e $\mathrm{VO}_{2}$ permanece constante. Em exercícios realizados nas intensidades do domínio pesado, o lactato e $\mathrm{o} \mathrm{VO}_{2}$ aumentam gradualmente, sem alcançar valores máximos. O domínio severo corresponde a intensidades de exercício próximas do $\mathrm{VO}_{2}$ máximo (CAMIC et al., 2010). Uma vez que essas intensidades marcam importantes diferenças em parâmetros metabólicos, o presente estudo almeja investigar se existe um comportamento análogo para variáveis biomecânicas - mais especificamente para a análise do sinal EMG e da AP e FP.

Características específicas nos testes incrementais, como alterações na duração de cada estágio incremental, diferentes formas de incremento da intensidade de exercício (velocidade ou inclinação) e modificações dos intervalos entre cada estágio incremental,

\section{Método}

\section{Amostra}

Participaram deste estudo 11 sujeitos que treinavam para competiçôes específicas de corrida. Foram adotados os seguintes critérios de inclusão: 1) todos os sujeitos deveriam apresentar frequência de treino de, no mínimo, três vezes por semana; 2) deveriam possuir experiência na modalidade superior a seis meses; e, 3) deveriam apresentar desempenho em provas com distância de $10 \mathrm{~km}$ em tempo inferior a 45 minutos. Foram excluídos da amostra os sujeitos que possuíam antecedentes de lesões osteomioarticulares nos membros inferiores e na coluna vertebral.

Todos os voluntários assinaram um termo de consentimento livre e esclarecido e foram orientados sobre as atividades que seriam realizadas durante $\mathrm{o}$ experimento. $\mathrm{O}$ desenho experimental do estudo foi podem influenciar os valores obtidos para cada limiar determinado. Na literatura, verificam-se estudos que compararam protocolos com diferentes duraçōes dos estágios de corrida incremental (FOXDAL, SJÖDIN \& Sjödin, 1995; Kuipers, Rietjens, Verstappen, SCHOENMARKERS \& HOFMAN, 2003), enquanto outros compararam protocolos com incrementos distintos velocidade e inclinação da esteira (KANG, CHALOUPKA, Mastrangelo, Biren \& Robertson, 2001). No entanto, faz-se necessária a realização de estudos que comparem os efeitos de diferentes intervalos entre os estágios de velocidade de protocolos incrementais de corrida sobre variáveis biomecânicas.

Diante do exposto, o objetivo do presente estudo é comparar o sinal EMG, a AP e a FP entre três diferentes intensidades de exercício, correspondentes a 60\%, 80\% e 100\% da velocidade máxima (Vmáx) obtida em dois diferentes protocolos incrementais de corrida.

Esse objetivo foi formulado a partir dos seguintes problemas de pesquisa: 1) o incremento na intensidade de corrida pode alterar o comportamento do sinal EMG, a AP e a FP?; 2) diferentes protocolos incrementais de corrida, que utilizam intervalos distintos entre cada estágio de velocidade, podem influenciar o comportamento do sinal EMG, a AP e a FP? As hipóteses desse estudo são que: 1) o sinal EMG, a AP e a FP são sensíveis às alterações na intensidade de corrida, apresentando aumento a partir do incremento da velocidade; 2 ) as variáveis analisadas sofrem influência do tipo de protocolo utilizado.

aprovado pelo Comitê de Ética em pesquisa local (Protocolo 2771/2004).

\section{Protocolo de teste}

Os sujeitos foram familiarizados na corrida em esteira com, pelo menos, 48 horas de antecedência ao protocolo de avaliação, durante nove minutos (LAVCANSKA, TAYLOR \& SCHACHE, 2005), divididos em estágios de três minutos nas velocidades de 10,11 e $12 \mathrm{~km} \cdot \mathrm{h}^{-1}$. Previamente à familiarização, foram realizadas as medidas antropométricas dos sujeitos avaliados para caracterização da amostra.

Os protocolos de corrida foram realizados com velocidade inicial de $10 \mathrm{~km} \cdot \mathrm{h}^{-1}$, com incrementos de $1 \mathrm{~km} \cdot \mathrm{h}^{-1}$ a cada três minutos até a exaustão voluntária. Para alcançar essa condição, foi fornecido estímulo verbal ao voluntário, sempre pelo mesmo avaliador. 
Os sujeitos estiveram no laboratório em dois dias para realização dos protocolos de corrida, com um intervalo mínimo de 48 horas entre eles, havendo diferença entre os protocolos somente no intervalo entre cada estágio de velocidade: intervalo de 30 segundos (protocolo A); intervalo de 120 segundos (protocolo B). A ordem de realização dos protocolos foi randomizada.

Os protocolos de corrida foram realizados em uma esteira ergométrica motorizada (Inbramed Super ATL - Brasil), com dimensões de $2 \mathrm{~m}$ de comprimento e $63 \mathrm{~cm}$ de largura.

A inclinação da esteira foi mantida fixa em $1 \%$, uma vez que tem esta condição reflete mais precisamente o custo energético da corrida em ambientes abertos (Jones \& DOUST, 1996).

Os sinais eletromiográficos e cinemáticos foram coletados durante todo o teste incremental. No final de cada estágio de velocidade de corrida foram coletadas amostras de $25 \mu \mathrm{l}$ de sangue do lóbulo da orelha, para posterior determinação de limares metabólicos, utilizados para caracterizar o grupo de sujeitos avaliados no presente estudo.

\section{Aquisição \\ e análise dos dados eletromiográficos}

Os sinais eletromiográficos foram registrados por meio de eletrodos de superfície bipolares descartáveis de $\mathrm{Ag} / \mathrm{AgCl}\left(\right.$ MediTrace $\left.^{\circledR}\right)$, com área de captação de $1 \mathrm{~cm}$ de diâmetro. Os eletrodos foram posicionados no lado direito do corpo, sobre os músculos iliocostal lombar (IC) no nível vertebral L2-L3 de acordo com Tsuboi, Satou, Egawa, IzUmi e Miyazaki (1994), reto femoral (RF), vasto lateral (VL), vasto medial (VM), bíceps femoral (porção longa) (BF), tibial anterior (TA), e gastrocnêmio (porção lateral) (GL), de acordo com SENIAM (Hermens, Freriks, Disselhorst-Klug \& RAU, 2002). A distância entre as regiōes centrais dos eletrodos foi de $2 \mathrm{~cm}$. O eletrodo de referência foi posicionado sobre a região anterior da tíbia.

Previamente à colocação dos eletrodos, foram realizados os procedimentos de tricotomia e limpeza da pele com lixa fina e álcool.

Para aquisição dos sinais EMG foi utilizado o sistema Telemyo 900 (Noraxon USA Inc., Scottsdale, AZ) e "software" Myoresearch (Version XP 1.6; Noraxon USA), com frequência de amostragem de $1000 \mathrm{~Hz}$ e com ganho de 2000 vezes ( 20 vezes no pré-amplificador e 100 vezes no condicionador de sinais). Foi utilizado um conversor analógico-digital (A/D) com resolução de 16 bits.

Para análise dos dados eletromiográficos, foi utilizada uma rotina desenvolvida em ambiente MatLab (versão
$6,5)$. Os dados brutos foram filtrados com filtro "notch" $60 \mathrm{~Hz}$, passa alta de $20 \mathrm{~Hz}$ e passa baixa de $500 \mathrm{~Hz}$.

Os sinais EMG foram retificados e analisados no domínio do tempo considerando a amplitude do sinal, expressa pelo do cálculo do valor RMS ("Root Mean Square").

Foi calculado um valor RMS para cada uma das últimas passadas, para que, posteriormente, fosse calculada a média desses valores em três diferentes intensidades de corrida, correspondentes a, aproximadamente, 60 , 80 e 100\% da velocidade máxima (Vmáx).

Previamente ao cálculo da média, os valores de RMS foram normalizados por um valor de referência comum correspondente ao valor de RMS da primeira passada, na velocidade inicial dos testes incrementais $\left(10 \mathrm{~km} \cdot \mathrm{h}^{-1}\right)$.

\section{Aquisição e análise dos dados cinemáticos}

Os dados cinemáticos foram coletados simultaneamente aos dados eletromiográficos, utilizando um módulo de sincronismo (sistema Telemyo 900 [Noraxon USA Inc., Scottsdale, AZ] e "software" Myoresearch [Version XP 1.6; Noraxon USA]).

Para captação das imagens foi utilizada uma filmadora digital (modelo NV-GS320, marca PANASONIC), que permitiu aquisição de dados em uma frequência de amostragem de $30 \mathrm{~Hz}$ e lâmpada refletora de $300 \mathrm{~W}$.

Posteriormente, essas imagens foram expostas a um procedimento de desentrelaçamento de quadros por meio do "software" Myoresearch (Version XP 1.6; Noraxon USA), o que permitiu a duplicação de sua frequência de amostragem $(60 \mathrm{~Hz})$.

Foi realizada análise bidimensional no plano sagital de movimento (cinemetria 2D), sendo que a câmera foi posicionada sobre um tripé a uma distância de 4,20 m da esteira, com uma altura de $90 \mathrm{~cm}$. Um quadro de calibração de dimensões conhecidas ( $1 \mathrm{mx} 1 \mathrm{~m}$ ) foi posicionado no início da filmagem, para definição da escala linear utilizada.

Um marcador reflexivo, com aproximadamente 2 $\mathrm{cm}^{2}$ foi posicionado no calcâneo direito. $\mathrm{O}$ ambiente de coleta foi preparado no intuito de assegurar a qualidade das imagens que foram, posteriormente, digitalizadas. Para tanto, o ambiente foi mantido escuro, utilizando exclusivamente a luminosidade proveniente da lâmpada refletora.

O "software" Peak Motus 9.0 (ViconPeak software) foi utilizado para digitalização automática e análise dos dados, o que permitiu o cálculo das coordenadas espaciais do ponto reflexivo do calcâneo. 
Após a digitalização da imagem, os dados foram expostos a um filtro do tipo Butterwoth de ordem quatro (Tillman, Hass, Chow \& Brunt, 2005), adotando-se uma frequência de corte de 6 $\mathrm{Hz}$, sendo, posteriormente, extraídas as variáveis correspondentes à AP e FP.

As 10 passadas analisadas foram divididas por sucessivos toques do calcâneo com o solo. Esses dados foram adquiridos por meio da análise cinemática que permitiu a obtenção dos dados referentes às coordenadas espaciais do ponto reflexivo localizado no calcâneo. Para localizar o ponto de contato do pé com o solo foram identificados os momentos em que maiores valores no eixo horizontal foram obtidos. O cálculo da AP foi realizado a partir das coordenadas referentes às distâncias percorridas pelo ponto do calcâneo no eixo horizontal (considerando os deslocamentos máximos e mínimos nesse eixo), conforme FIGURA 1 e a equação que segue.

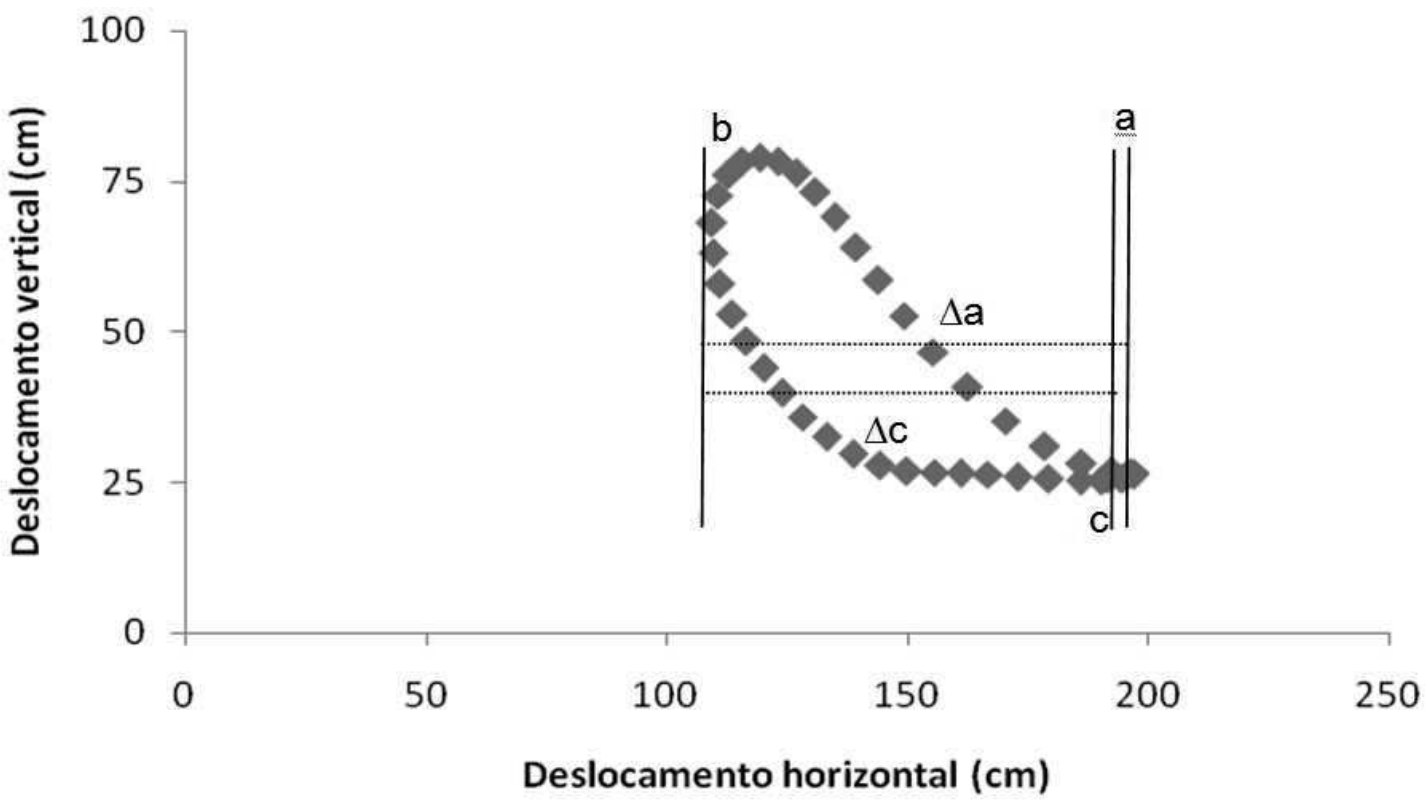

FIGURA 1 - Deslocamentos verticais e horizontais a partir do eixo de coordenadas do ponto do calcâneo, representativo de um ciclo de passada.

Para cálculo da AP foi utilizada a seguinte equação:

$$
\mathrm{AP}=\Delta \mathrm{ab}+\Delta \mathrm{cb}
$$

Onde,

$\mathrm{AP}=$ amplitude de passada.

$\mathrm{a}=$ valor máximo da coordenada horizontal obtido no contato do calcâneo com o solo.

$\mathrm{b}=$ valor mínimo da coordenada horizontal, referente à fase de voo.

$\mathrm{c}=$ valor máximo da coordenada horizontal obtido no contato subsequente do calcâneo com o solo.

$$
\begin{aligned}
\Delta \mathrm{ab} & =\mathrm{a}-\mathrm{b} \\
\Delta \mathrm{cb} & =\mathrm{c}-\mathrm{b}
\end{aligned}
$$

A FP foi calculada a partir da duração de contatos sucessivos do calcâneo com o solo.

De forma idêntica à análise do sinal EMG, a AP e a FP foram calculadas considerando a média das últimas passadas, e comparadas nas intensidades aproximadas de 60, 80 e 100\% da velocidade máxima (Vmáx).

\section{Aquisição \\ e análise das amostras sanguíneas}

Previamente a coleta de sangue, foi realizada assepsia local com álcool e algodão e a punção do lóbulo da orelha com uma lanceta descartável. A primeira gota de sangue foi descartada para evitar a contaminação da gota de sangue pelo suor produzido.

Foram coletados $25 \mu \mathrm{l}$ de sangue arterializado do lóbulo da orelha com capilares de vidro heparinizados e calibrados.

Após cada coleta, o sangue foi imediatamente depositado em tubos tipo "eppendorf" de $1,5 \mathrm{ml}$ contendo $50 \mu \mathrm{l}$ de fluoreto de sódio a $1 \%$.

O lactato sanguíneo foi determinado por um método eletroquímico (YSL 2300 STAT, Yelow Springs, Ohio, USA).

O Limiar de Lactato (LL) foi calculado por meio do ponto de inflexão na curva lactato-intensidade 
(determinado por três pesquisadores experientes), e o Limiar Anaeróbio (LAn) foi determinado por meio de interpolação linear, considerando a concentração fixa de 3,5 mM (Heck, MAdER, Hess, Mucke, Muller \& Hollmann, 1985).

\section{Análise estatística}

Os valores de RMS, de AP e FP foram expressos em médias e desvios-padrão em cada protocolo, sendo verificada a normalidade da distribuição dos dados utilizando o teste de Shapiro-Wilk.
Foi utilizado o teste ANOVA - "one way" para medidas repetidas, com teste "post hoc" de Bonferroni, para comparação dos valores de RMS, AP e FP entre as intensidades correspondentes a 60, 80 e 100\% da Vmáx obtida no protocolo incremental de corrida.

Para comparação dos valores de RMS e das variáveis cinemáticas entre os dois protocolos de teste foi aplicado um teste $\mathrm{t}$ de Student para dados pareados.

Foi considerado, para todos os cálculos, $\mathrm{p}<0,05$. Para a realização do tratamento estatístico foi utilizado o aplicativo SPSS (Statistical Package for Social Sciences), (versão 13.0, Chicago, USA).

\section{Resultados}

$\mathrm{Na}$ TABELA 1 são apresentados valores referentes à idade e aos dados antropométricos dos sujeitos que compóem a amostra deste estudo, bem como os seus respectivos valores de média e desvio-padrão.

A TABELA 2 mostra os valores correspondentes às intensidades de 60, 80 e 100\% da Vmáx, e os valores referentes aos limiares metabólicos (LAn e LL), nos protocolos A e B.

TABELA 1 - Características amostrais correspondentes à idade e aos dados antropométricos dos sujeitos que compõem a amostra deste estudo, e aos seus respectivos valores de média e desvio-padrão.

\begin{tabular}{ccccc}
\hline Sujeitos & Idade (anos) & Peso $(\mathbf{k g})$ & Altura $(\mathbf{m})$ & \% de gordura \\
\hline 1 & 27 & 53 & 1,7 & 9 \\
2 & 26 & 64,5 & 1,79 & 8 \\
3 & 25 & 84,5 & 1,8 & 12 \\
4 & 19 & 59,5 & 1,7 & 7 \\
5 & 18 & 61 & 1,75 & 8 \\
6 & 19 & 71,5 & 1,75 & 10 \\
7 & 36 & 80 & 1,8 & 13 \\
8 & 31 & 83 & 1,83 & 12 \\
9 & 19 & 60 & 1,78 & 7 \\
10 & 28 & 86,5 & 1,84 & 13 \\
11 & 18 & 69,5 & 1,81 & 8 \\
\hline Média & $\mathbf{2 4 , 2}$ & $\mathbf{7 0 , 3}$ & $\mathbf{1 , 7 8}$ & $\mathbf{9 , 7}$ \\
DP & $\mathbf{6 , 1}$ & $\mathbf{1 1 , 7}$ & $\mathbf{0 , 0 5}$ & $\mathbf{2 , 4}$ \\
\hline
\end{tabular}

TABELA 2 - Velocidades $\left(\mathrm{km} . \mathrm{h}^{-1}\right)$, expressas em média \pm desvio-padrão, correspondentes às intensidades de 60, 80 e 100\% da Vmáx, LAn e LL nos protocolos A e B.

\begin{tabular}{lccccc}
\hline \multicolumn{5}{c}{ Velocidade $\left(\mathrm{km} \cdot \mathrm{h}^{-1}\right)$} \\
\hline Protocolo A & $60 \%$ da Vmáx & $80 \%$ da Vmáx & $100 \%$ da Vmáx & LL & LAn \\
Protocolo B & $10,5 \pm 0,4$ & $13,6 \pm 1,1$ & $17,0 \pm 1,3$ & $12,8 \pm 1,0$ & $14,9 \pm 1,7$ \\
\hline
\end{tabular}


Foi possível verificar que nos dois protocolos testados, a velocidade aproximada a $60 \%$ da Vmáx foi inferior à intensidade correspondente ao LL, e a velocidade aproximada a $80 \%$ foi inferior à intensidade correspondente ao LAn. Parâmetros metabólicos como LL e LAn são utilizados na literatura para demarcar as intensidades de exercício moderado e pesado, respectivamente. Diante disto, a TABELA 2 confirma que as intensidades relativas a Vmáx se encontram, aproximadamente, nos diferentes domínios propostos.

As FIGURAS 2 e 3 apresentam a comparação do valor RMS nas três diferentes intensidades de corrida nos dois protocolos de teste.
${ }^{*} p<0,05$ diferença significativa de $60 \%$;

$\# p<0,05$ diferença significativa de $80 \%$.
* $p<0,05$ diferença significativa de $60 \%$;

$\# p<0,05$ diferença significativa de $80 \%$.

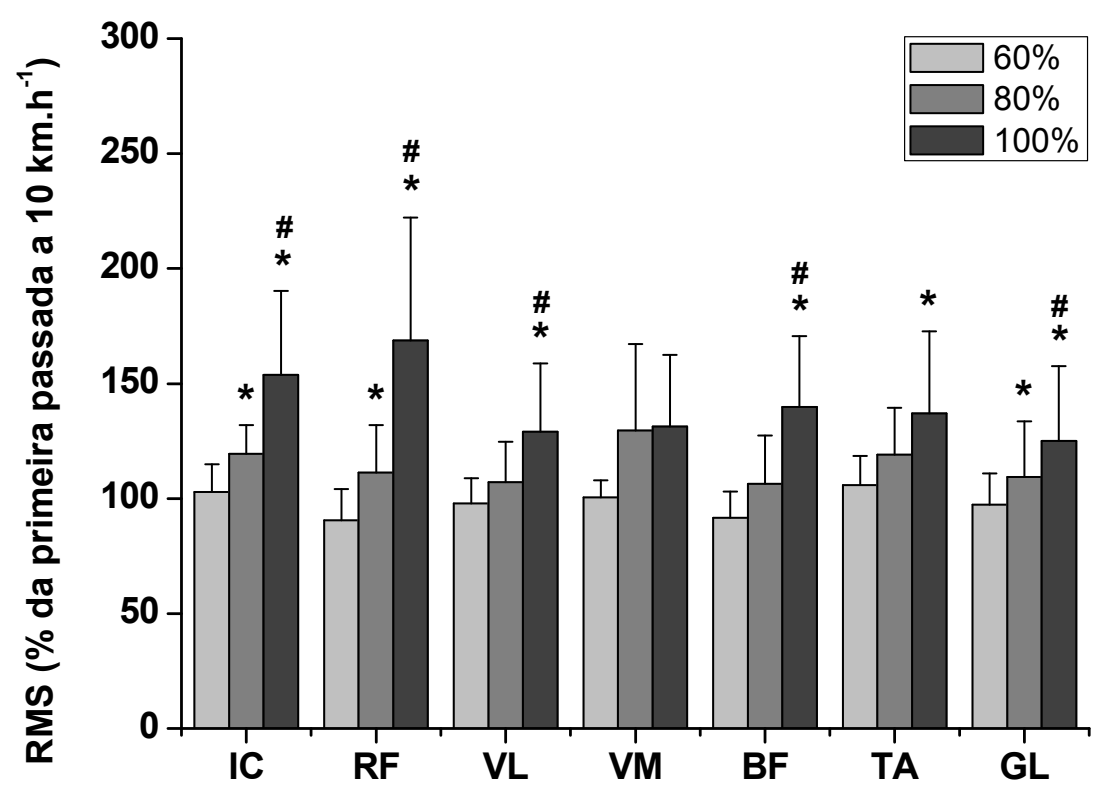

FIGURA 2 - Comparação entre as intensidades de corrida correspondentes a 60, 80 e 100\% da Vmáx, considerando os valores RMS (média \pm DP) dos músculos IC $(n=10), R F(n=11), V L(n=11), V M(n=7), B F(n=10)$, $\mathrm{TA}(\mathrm{n}=10)$ e GL $(\mathrm{n}=11)$ normalizados pelo valor de RMS da primeira passada a $10 \mathrm{~km} . \mathrm{h}^{-1}$, no protocolo A.

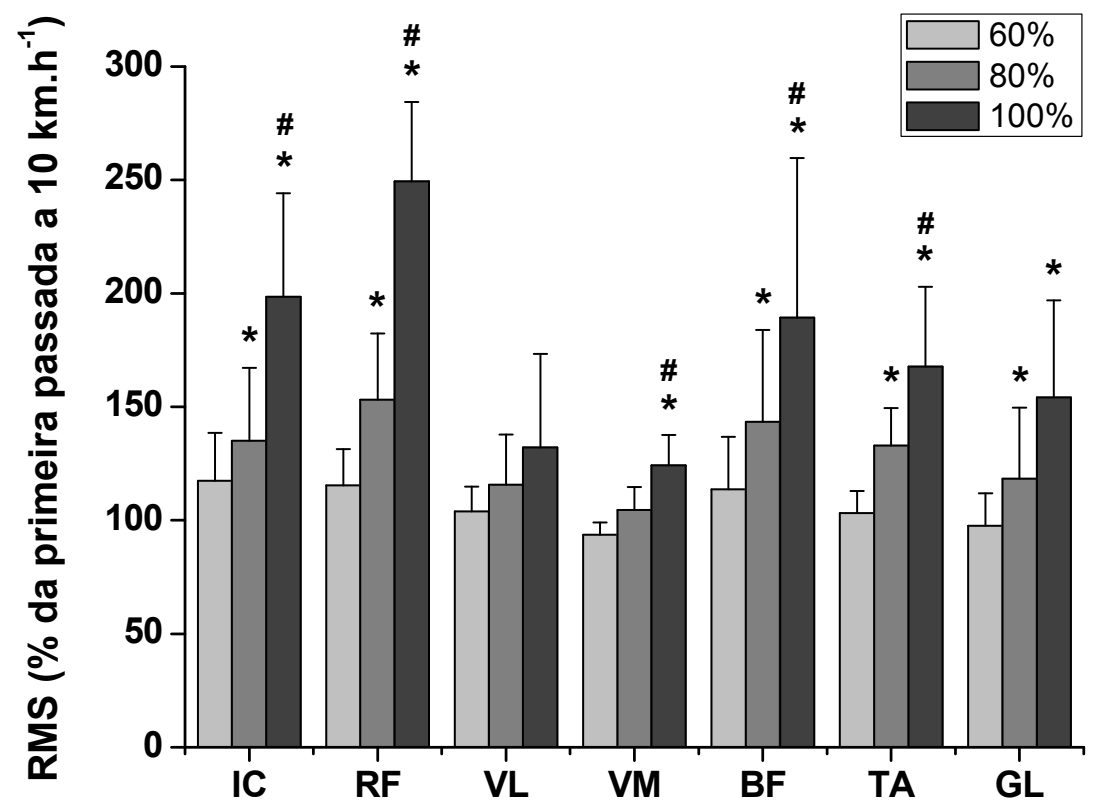

FIGURA 3 - Comparação entre as intensidades de corrida correspondentes a 60, 80 e 100\% da Vmáx, considerando os valores RMS (média \pm DP) dos músculos IC $(n=7), \operatorname{RF}(n=8), V L(n=11), V M(n=8), B F(n=11)$, TA $(n=9)$ e GL $(n=8)$ normalizados valor de RMS da primeira passada a $10 \mathrm{~km} . \mathrm{h}^{-1}$ no protocolo $\mathrm{B}$. 
Foi possível observar que, quando os valores de RMS foram comparados entre diferentes velocidades de corrida, esses mostraram aumentos significativos entre as diferentes intensidades $(60,80 \mathrm{e}$ 100\% da Vmáx.), para quase todos os músculos analisados, exceto para os músculos VM (protocolo A) e VL (protocolo B).
Dessa forma, similar aos parâmetros metabólicos (LL e LAn), foi verificado que os valores de RMS mostraramse sensíveis às alterações das intensidades correspondentes aos domínios moderado, pesado e severo.

A TABELA 3 apresenta a comparação entre os protocolos de teste utilizados (Protocolo A e B) a partir dos valores de RMS.

TABELA 3 - Comparação entre os protocolos de teste utilizados (Protocolo A e B), nas intensidades de corrida correspondentes a 60, 80 e 100\% da Vmáx, considerando os valores RMS (média \pm DP) dos músculos IC, RF, VL, VM, BF, TA e GL normalizados pelo valor de RMS da primeira passada (10 $\left.\mathrm{km} \cdot \mathrm{h}^{-1}\right)$

\begin{tabular}{lcccccc}
\hline \multicolumn{7}{c}{ R0\% } \\
\hline & Protocolo A & Protocolo B & Protocolo A & Protocolo B & Protocolo A & Protocolo B \\
\hline IC & $102,9 \pm 12,0$ & $117,5 \pm 21,1$ & $119,5 \pm 12,4$ & $135,0 \pm 32,2$ & $153,8 \pm 36,5$ & $198,5 \pm 45,7$ \\
RF & $90,6 \pm 13,5 \Delta$ & $115,3 \pm 16,0$ & $111,2 \pm 20,8 \Delta$ & $153,2 \pm 29,2$ & $168,7 \pm 53,5 \Delta$ & $249,4 \pm 35,0$ \\
VL & $97,9 \pm 11,0$ & $103,8 \pm 11,1$ & $107,0 \pm 17,6$ & $115,8 \pm 22,1$ & $129,0 \pm 29,8$ & $132,1 \pm 41,2$ \\
VM & $100,5 \pm 7,5$ & $93,6 \pm 5,5$ & $129,5 \pm 37,7$ & $104,6 \pm 10,1$ & $131,3 \pm 31,1$ & $124,3 \pm 13,4$ \\
BF & $91,6 \pm 11,4 \Delta$ & $113,6 \pm 23,2$ & $106,4 \pm 21,1 \Delta$ & $143,4 \pm 40,6$ & $139,8 \pm 30,9$ & $189,4 \pm 70,3$ \\
TA & $105,8 \pm 12,7$ & $103,2 \pm 9,7$ & $119,1 \pm 20,3$ & $133,0 \pm 16,4$ & $137,1 \pm 35,7$ & $167,7 \pm 35,2$ \\
GL & $97,3 \pm 13,6$ & $97,7 \pm 14,2$ & $109,4 \pm 24,2$ & $118,4 \pm 31,3$ & $125,0 \pm 32,6$ & $154,2 \pm 42,7$ \\
\hline
\end{tabular}

$\Delta p<0,05$ diferença significativa em relação ao Protocolo B.
Foram verificados aumentos significativos do protocolo A para o protocolo $\mathrm{B}$, apenas para o músculo RF [60\% (p = 0,012), 80\% (p = 0,000) e 100\% da Vmáx ( $\mathrm{p}=0,003)]$; e para o músculo BF $[60 \%$ (p $=0,045)$ e $80 \%$ da Vmáx $(\mathrm{p}=0,011)]$.

Dessa forma, o valor RMS não foi influenciado pelo tipo de protocolo utilizado para maioria dos músculos analisados no presente estudo, exceto para os músculos bi-articulares RF e BF.

A FIGURA 4 apresenta o comportamento das variáveis cinemáticas (AP e FP), nas diferentes intensidades de corrida, para os dois grupos avaliados nos dois diferentes protocolos de teste.

Quando comparadas a AP e FP entre as diferentes intensidades de corrida foi verificado que as duas variáveis aumentam de forma significativa concomitantemente com o incremento da velocidade. Esse resultado era esperado, uma vez que a velocidade é determinada pelo produto direto entre essas duas variáveis.

Entretanto, ocorre um aumento percentual distinto dessas variáveis de $60 \%$ para $100 \%$ da Vmáx: a AP aumenta 27,8\% no Protocolo A, e 29,0\% no Protocolo B; enquanto que a FP aumenta $8,5 \%$ no Protocolo A e $12 \%$ no Protocolo B. Com isso, pode-se inferir que essas variáveis apresentam uma contribuição percentual diferenciada para aumento a velocidade, sendo que a AP apresenta maior contribuição percentual.

Quando comparados os diferentes protocolos de teste, foi verificado que AP mostrou-se significativamente maior no protocolo $\mathrm{B}$ do que no protocolo $\mathrm{A}$ em 80\% da Vmáx. ( $\mathrm{p}=0,043)$ e 100\% da Vmáx. ( $\mathrm{p}=$ 0,040); e FP também foi maior no protocolo B quando comparado ao protocolo A em $80 \%$ da Vmáx. ( $\mathrm{p}=$ 0,007) e 100\% da Vmáx. ( $p=0,004)$, (TABELA 4).

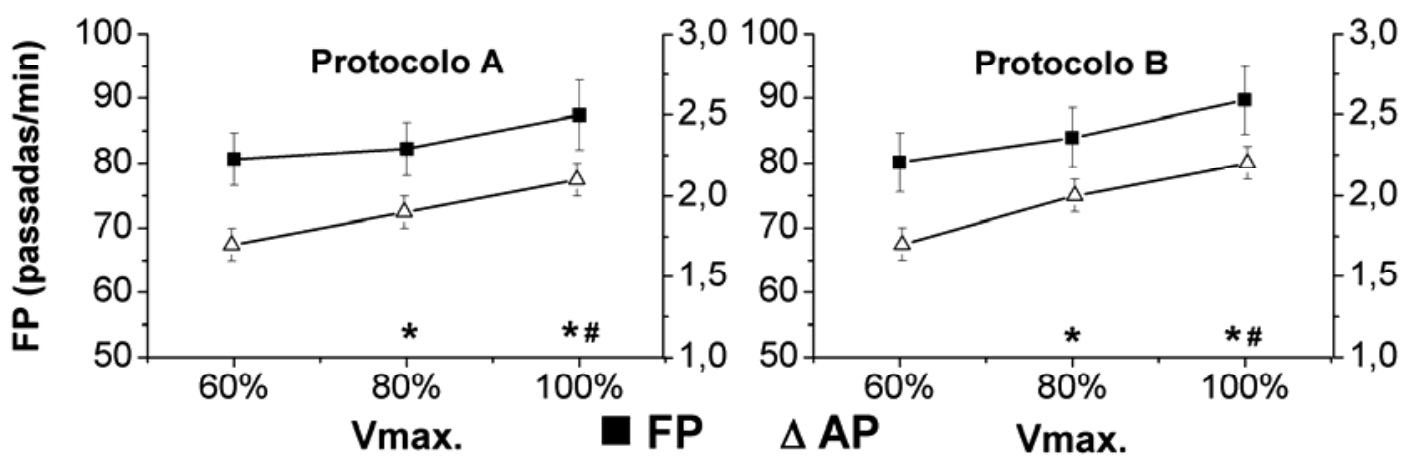

${ }^{*} p<0,05$ indica diferença significativa de $60 \%$ para AP e FP; \# $p<0,05$ indica diferença significativa de $80 \%$ para AP e FP.

FIGURA 4 - Comparação daAPeFP(média \pm DP) entreas intensidades decorrida correspondentesa 60, 80e100\% da Vmáx. 
TABELA 4 - Comparação entre os grupos avaliados (CR e CO) e entre os protocolos de teste utilizados (Protocolo Ae B), nas intensidades de corrida correspondentes a 60, 80 e 100\% da Vmáx, considerando os valores de AP FP.

$\Delta p<0,05$ diferença significativa em relação ao Protocolo B.

\begin{tabular}{ccccccc}
\hline \multicolumn{7}{c}{ Variáveis Cinemáticas } \\
\hline & Protocolo A & Protocolo B & Protocolo A & Protocolo B & Protocolo A & Protocolo B \\
\hline FP & $80,6 \pm 4,0$ & $80,1 \pm 4,5$ & $82,2 \pm 4,1 \Delta$ & $83,9 \pm 4,6$ & $87,4 \pm 5,4 \Delta$ & $89,7 \pm 5,3$ \\
AP & $1,66 \pm 0,1$ & $1,68 \pm 0,1$ & $1,91 \pm 0,1 \Delta$ & $1,97 \pm 0,1$ & $2,12 \pm 0,1 \Delta$ & $2,17 \pm 0,1$ \\
\hline
\end{tabular}

\section{Discussão}

O presente estudo teve por objetivo comparar o sinal eletromiográfico, a AP e FP em três diferentes intensidades de exercício durante dois protocolos de corrida incrementais.

Foi verificado que os valores de RMS mostraramse sensíveis às alteraçōes das intensidades correspondentes a 60, 80 e 100\% da Vmáx., de forma semelhante ao que ocorre com os parâmetros metabólicos (CAmic et al., 2000).

O comportamento do valor de RMS para maioria dos músculos analisados foi de aumento como resultado do incremento velocidade; exceto para os músculos VM (protocolo A) e VL (protocolo B), que não alteraram significativamente sua ativação a partir de uma maior intensidade de teste.

Entretanto, embora seja possível observar o referido aumento do valor RMS como resposta ao incremento da intensidade de corrida para diferentes músculos na maioria das condições analisadas, apenas os músculos IC e RF apresentaram aumentos entre todas as intensidades, nos dois protocolos de teste utilizados.

GAZENDAM e Hof (2007) afirmam que não há na corrida um comportamento estável do sinal EMG para diferentes músculos em resposta a um protocolo incremental de corrida: o sinal EMG pode aumentar, permanecer constante ou, até mesmo, diminuir com o incremento de velocidade, dependendo do músculo a ser analisado. GAZENDAM e HoF (2007) verificaram que a amplitude do sinal EMG do músculo BF aumentou com o incremento de velocidade de corrida até a velocidade de 3,0 m.s. reduzindo após essa velocidade até $4,5 \mathrm{~m} \cdot \mathrm{s}^{-1}$.

Outros estudos têm relatado comportamentos diversificados de ativação muscular. Enquanto GUIDETTI, RIVELLINI e FiguRA (1996) afirmam que o músculo RF apresenta dois períodos de ativação em um ciclo, GAZENDAM e Hof (2007) apontam que esse músculo, embora biarticular, teria ativação apenas em uma fase do ciclo de passada (correspondente a fase de contato).
Avogadro, Dolenec e Belu (2003) também relatam adaptaçôes específicas para os diferentes músculos analisados, pois verificaram aumento da atividade somente para o músculo VL quando comparado o período inicial e final de uma corrida prolongada. Neste caso, foi considerado como período inicial aquele correspondente ao terceiro minuto de corrida e o período final foi correspondente à coleta realizada antes da exaustão em um protocolo com intensidade constante de $90 \%$ do $\mathrm{VO}_{2 \text { maxx }}$. Não foram encontradas diferenças significativas entre os demais músculos estudados (BF e TA).

Diante disso, a padronização da atividade muscular a partir de corridas incrementais que induzam a fadiga deve ser realizada com cautela. Diferentemente do ciclismo, que envolve ciclos de pedalada dentro de uma forma de movimento mais previsível, a corrida permite uma série de ajustes na passada (por exemplo, na AP e FP) que podem alterar substancialmente a amplitude do sinal EMG (HANON, Thépaut-Mathieu \& Vandewalle, 2005).

Os protocolos incrementais utilizados diferiram quanto ao intervalo entre cada estágio de velocidade. Diferente do ciclismo, em que os protocolos incrementais para determinação de limiares metabólicos não exigem que o atleta interrompa a atividade para coletas de amostras sanguíneas, para determinação de limiares metabólicos durante a corrida são utilizados protocolos incrementais com o menor intervalo possível entre cada estágio, sendo utilizado apenas o tempo suficiente para adequada coleta dessas amostras. Dessa forma, geralmente, são utilizados protocolos com intervalos de 30 segundos. Entretanto, o presente estudo comparou as variáveis obtidas em protocolos incrementais com intervalos maiores (120 s), no intuito de observar se o maior tempo entre os estágios promove alguma recuperação capaz de alterar as características das variáveis biomecânicas que foram analisadas.

Além disso, essa comparação torna-se pertinente, pois a falta de padronização nas características 
específicas dos testes incrementais de corrida (tais como duração dos estágios incrementais e duração entre os incrementos) pode dificultar a comparação entre diferentes estudos.

Foi verificado que o comportamento do valor RMS não foi influenciado pelo tipo de protocolo utilizado para maioria dos músculos analisados, exceto para os músculos biarticulares RF e BF.

Diversos estudos relatam que existem peculiaridades que diferem a atuação de músculos mono e biarticulares (DoORenbosch \& VAN Ingen SCHENAU, 1995; Hanon, Thépaut-Mathieu \& Vandewalle, 2005; Van Ingen Schenau, Pratt \& MacPherson, 1994).

De acordo com Doorenbosch e Van Ingen Schenau (1995) e Van Ingen Schenau, Pratt e MacPherson (1994), músculos monoarticulares apresentam importante papel na produção de contraçōes concêntricas, enquanto que os músculos biarticulares fornecem uma fina regulação na distribuição dos momentos sobre as articulações.

Especula-se que a ativação muscular pode ser controlada a partir de um critério de prevalência. Quando uma tarefa requer certa distribuição dos momentos independente do comprimento muscular, os músculos biarticulares são ativados. Por outro lado, quando um grande trabalho é realizado durante uma tarefa particular, a contribuição dos músculos monoarticulares é mais pronunciada (DoORENBOSCH \& Van Ingen SCHENAU, 1995; Hanon, Thépaut-Mathieu \& Vandewalle, 2005).

Para Kyröläinen, Avela e Komi (2005), o BF tende a apresentar sua principal contribuição na corrida na potente extensão do quadril, o que the confere grande importância na fase de propulsão do movimento. Além disso, esse músculo atua excentricamente durante a fase aérea para armazenar energia elástica de forma a favorecer a fase propulsora subsequente. Assim, os ísquios-tibiais são potencialmente ativados para aumentar a força propulsiva gerando maior força resultante no sentido horizontal.

VuorimaA, Virlander, Kurkilahti, Vasankari e HÃKKINEN (2006) verificaram que após corridas intensas os músculos agonistas e antagonistas ao movimento de extensão do joelho foram ativados diferentemente: foi observada redução do sinal EMG dos músculos VL e $\mathrm{VM}$, enquanto que não foram encontradas diferenças significativas no sinal EMG do músculo BF.

Considerando a análise do comportamento da passada, por meio da análise das variáveis cinemáticas, o presente estudo evidenciou aumento significativo dos valores correspondentes a AP e FP entre todas as intensidades comparadas. Esse resultado mostrava-se bastante previsível, uma vez que mudanças na velocidade de corrida são acompanhadas por alterações na AP e FP (CAPPELlini, IVANENKo, PopPele \& LaCQuaniti, 2006).

A AP é determinada pela soma do deslocamento horizontal do corredor durante a fase de apoio da passada e na fase de balanço. A FP corresponde ao número de passadas por unidade de tempo (CAVANAGH \& KRAM, 1989).

Entretanto, parece não haver consenso na literatura sobre o percentual de contribuição de cada uma dessas variáveis na determinação da velocidade (Bus, 2003; Elliot \& Ackland, 1981; Martin \& SANDERSON, 2000). O presente estudo evidenciou que a AP possui maior contribuição percentual do que a FP para o aumento da velocidade em um teste incremental de corrida, o que corrobora com os resultados encontrados por Bus (2003).

$\mathrm{Na}$ comparação das variáveis cinemáticas entre os protocolos de teste distintos, foram observadas diferenças significativas na AP e na FP nas intensidades de 80 e 100\% da Vmáx. Com isso, sugere-se que alteraçóes no intervalo entre os estágios incrementais modificaram o comportamento da passada no protocolo B em que velocidades absolutas maiores foram atingidas, o que, possivelmente esteja relacionado aos referidos aumentos nas variáveis cinemáticas.

De acordo com os resultados apresentados, pode-se inferir que, semelhante às variáveis metabólicas, o sinal EMG dos músculos analisados mostrou-se sensível às alteraçôes na intensidade de corrida quando comparadas 60, 80 e 100\% da Vmáx. Dessa forma, sugere-se que mais estudos sejam realizados sobre esse assunto na tentativa de propor limiares obtidos para músculos individuais (como, por exemplo, limiares eletromiográficos), os quais podem ser comparados aos limiares metabólicos já comumente utilizados na prescrição de treinamentos. Isso permitirá avaliar se esses limiares eletromiográficos mostram-se semelhantes aos limiares sistêmicos determinados a partir de protocolos incrementais de corrida. Além disso, os limiares eletromiográficos dos músculos individuais podem, eventualmente, indicar quais músculos podem estar restringindo rendimentos melhores em cada fase específica de treinamento. A identificação de músculos que estejam sofrendo fadiga precocemente pode ser útil para direcionar o treinamento visando atender a esta característica do músculo na execução da corrida. Vale lembrar, contudo, que este objetivo é bastante audacioso e torna-se necessário determinar anteriormente as características da variável Limiar Eletromiográfico nas mais diversas condições para que ela seja utilizada com esta finalidade. 
Os resultados aqui apresentados mostraram que o comportamento do valor RMS para a maioria dos músculos analisados não foi influenciado pelo tipo de protocolo utilizado. A principal implicação deste resultado é que intervalos maiores entre os estágios de um protocolo de corrida incremental podem ser adotados sem alterar o comportamento do sinal EMG, o que possibilita a coleta de dados adicionais durante tais intervalos, como, por exemplo, coleta de contraçóes isométricas nesses intervalos. Entretanto, sugere-se que mais estudos sejam realizados comparando o sinal EMG em diferentes protocolos.

Já as variáveis cinemáticas sofreram influência em função do protocolo utilizado. Dentre as variáveis analisadas, a AP apresentou maior contribuiçãao percentual para aumento da velocidade de corrida do que a FP. Sugere-se que mais estudos sejam realizados comparando essas contribuições percentuais da AP e da FP no aumento da velocidade entre corredores experientes e iniciantes na modalidade.
Sumarizando, o presente estudo mostrou que, quando comparadas diferentes intensidades de um protocolo incremental de corrida $(60,80$ e $100 \%$ da Vmáx), o sinal EMG tende a apresentar aumento concomitante ao incremento de velocidade. Entretanto, não foi verificado um comportamento característico para os músculos analisados nesse estudo. Além disso, o aumento da velocidade de corrida ocorreu tanto pelo aumento da FP, como pelo aumento da AP, embora essas variáveis tendam a apresentar contribuiçôes percentuais diferenciadas com o incremento de velocidade. Protocolos de teste com intervalos distintos entre os estágios incrementais não alteraram o comportamento do valor RMS para maioria dos músculos analisados, exceto para os músculos biarticulares RF e BF. Além disso, no protocolo com maior intervalo de recuperação (protocolo $\mathrm{B}$ ), as variáveis cinemáticas $\mathrm{AP}$ e FP foram maiores do que no protocolo A nas intensidades de 80 e $100 \%$ da Vmáx.

\section{Abstract \\ Comparison of EMG activity and step characteristics of different running incremental protocols}

The aim of this study was to compare the electromyographic signal and the stride frequency and length among different intensities of running: 60\%, 80\% and 100\% of maximum speed in two incremental protocols. The study included 11 male runners. The protocols were performed with initial running speed of $10 \mathrm{~km} \cdot \mathrm{h}^{-1}$, with increments of $1 \mathrm{~km} \cdot \mathrm{h}^{-1}$ at every three minutes until exhaustion. The two protocols differed in the interval between each incremental stage: 30 to 120 seconds. We analyzed RMS values of the iliocostalis lumbar, rectus femoris, vastus lateralis, vastus medialis, biceps femoris, tibialis anterior, gastrocnemius lateralis, and the stride length and frequency. The RMS values showed an increase among intensities for almost all muscles, and were not influenced by the type of protocol used for most muscles. The contribution of stride length and frequency were percentile different as the running speed increased.

UNITERMS: Electromyography; Kinematics; Runners.

\section{Resumen}

Comparación de la actividad EMG y caracteristicas del pasado en los diferentes protocolos de raza incrementales

El objetivo de este estudio es comparar la actividad electromiográfica, la amplitud y la frecuencia del pasado entre las diferentes intensidades de carrera: 60\%, 80\% y 100\% de la velocidad máxima en dos protocolos adicionales. En el estudio participaron 11 corredores. Los protocolos se realizaron con velocidades de carrera iniciales de $10 \mathrm{~km} \cdot \mathrm{h}^{-1}$, con incrementos de $1 \mathrm{~km} \cdot \mathrm{h}^{-1}$ cada tres minutos hasta que se agoten, que diferian en el intervalo entre cada etapa incremental: 30 a 120 segundos. Se analizaron los valores RMS de las iliocostalis lumbar, rectus femoris, vastus lateralis, vastus medialis, biceps femoris, tibialis anterior, gastrocnemius, la amplitud y la frecuencia del pasado. Los valores de RMS mostró un aumento entre las intensidades para casi 
todos los músculos, y no fueron influenciadas por el tipo de protocolo utilizado. Las contribuciones de frecuencia y amplitud de pasado presentaran porcentaje diferenciado para aumentar la velocidad de funcionamiento.

PALABRAS ClAVE: La electromiografía; Cinemática; Corredores.

\section{Referências}

ABE, D.; MURAKI, S.; YANAGAWA, K.; FUKUOKA, Y.; NIIHATA, S. Changes in EMG characteristics and metabolic energy cost during 90-min prolonged running. Gait and Posture, Oxford, v.26, p.607-10, 2007.

AVOGRADO, P.; DOLENEC, A.; BELLI, A. Changes in mechanical work during severe exhausting running. European Journal of Applied Physiology, Berlin, v.90, p.165-70, 2003.

BASMAJIAN, J.V.; DE LUCA, C.J. Muscle alive: their functions revealed by electromyography, 5. ed. Baltimore/Maryland: Williams \& Wilkins, 1985.

BUS, S.A. Ground reaction forces and kinematics in distance running in older-aged men. Medicine and Science in Sports and Exercise, Philadelphia, v.35, n.7, p.1167-75, 2003.

CAMIC, C.L.; HOUSH, T.J.; JOHNSON, G.O.; HENDRIX, C.R.; ZUNIGA, J.M.; MIELKE, M.; SCHMIDT, R.J. An EMG frequency-based test for estimating the neuromuscular fatigue threshold during cycle ergometry. European Journal of Applied Physiology, Berlin, v.108, n.2, p.337-45, 2010.

CAPPELLINI, G.; IVANENKO, Y.P.; POPPELE, R.E.; LACQUANITI, F. Motor patterns in human walking and running. Journal of Neurophysiology, Bethesda, v.95, p.3426-437, 2006.

CARTER, H.; JONES, A.M.; DOUST, J.M. Changes in blood lactate and pyruvate concentrations and the lactate-topyruvate ratio during the lactate minimum speed test. Journal of Sports Science, London, v.18, p.213-25, 2000.

CARTER, H.; PRINGLE, J.S.M.; JONES, A.M.; DOUST, J.M. Oxygen uptake kinetics during treadmill running across exercise intensity domains. European Journal of Applied Physiology, Berlin, v.86, p.347-54, 2002.

CAVANAGH, P.R.; KRAM, R. Stride length in distance running: velocity, body dimensions, and added mass effects. Medicine and Science in Sports and Exercise, Philadelphia, v.21, n.4, p. 467-79, 1989.

DOORENBOSCH, C.A.M.; VAN INGEN SCHENAU, G.J.I. The role of mono- and biarticular muscles during control leg tasks in man. Human Movement Science, Amsterdam, v.14, p.279-300, 1995.

ELLIOT, B.; ACKLAND, T. Biomechanical Effects of Fatigue on 10.000 Meter Running Technique. Research Quarterly for Exercise and Sport, Reston, v.52, n.2, p.160-66, 1981.

FOXDAL, P.; SJÖDIN, A.; SJÖDIN, B. Comparison of blood lactate concentration obtained during incremental and constant intensity exercise. International Journal of Sports Medicine, Stuttgart, v.17, p.360-365, 1995.

FRAGA, C.H.W.; BLOEDOW, L.S.; GUIMARÃES, A.C.S.; VAZ, M.A. Análise de variáveis cinemáticas na corrida do triathlon obtidas em prova simulada. Revista Brasileira de Biomecânica, São Paulo, v.8, n.14, p.16-20, 2007.

FRAGA, C.H.W.; SILVA, S.R.; GONÇALVES, M. Effect of running velocity on electromyographic and metabolic variables. Motriz, Rio Claro, v.15, p.911-18, 2009.

GAZENDAM, M.G.J.; HOF, A.L. Averaged EMG profiles in jogging and running at different speeds. Gait and Posture, Oxford, v.25, p.604-14, 2007.

GUIDETTI, L.; RIVELLINI, G.; FIGURA F. EMG Patterns During Running: Intra- and Inter-individual Variability. Journal of Electromyography and Kinesiology, Oxford, v.6, p.37-48, 1996.

HANON, C.; THÉPAUT-MATHIEU, C.; HAUSSWIRTH, C.; LE CHEVALIER, J.M. Electromyogram as an indicator of neuromuscular fatigue during incremental exercise. European Journal of Applied Physiology and Occupational Physiology, Berlin, v.78, p.315-23, 1998.

HANON, C.; THÉPAUT-MATHIEU, C.; VANDEWALLE, H. Determination of muscular fatigue in elite runners. European Journal of Applied Physiology, Berlin, v.94, p.118-25, 2005.

HECK, H.; MADER, A.; HESS, G.; MUCKE, S.; MULLER, R.; HOLLMANN, W. Justification of the $4 \mathrm{mmol} / \mathrm{l}$ lactate threshold. International Journal of Sports Medicine, Stuttgart, v.6, p.117-30, 1985.

HERMENS, H.J.; FRERIKS, B.; DISSELHORST-KLUG, C.; RAU, G. The SENIAM project: surface electromyography for non-invasive assessment of muscle. In: CONGRESS OF THE INTERNATIONAL SOCIETY OF ELECTROPHYSIOLOGY AND KINESIOLOGY, 14., Vienna, 2002. Proceedings... Vienna: ISEK, 2002. [CD-ROM]. 
JONES, A.M.; DOUST, J.H. A 1\% treadmill grade most accurately reflects the energetic cost of outdoor running. Journal of Sport Science, London, v.14, p.321-27, 1996.

KANG, J.; CHALOUPKA, E.C.; MASTRANGELO, M.A.; BIREN, G.B.; ROBERTSON, R.J. Physiological comparisons among three maximal treadmill exercises protocols in trained and untrained individuals. European Journal of Applied Physiology, Berlin, v.84, p.291-95, 2001.

KARAMANIDIS, K.; ARAMPATZIS, A.; BRÜGGEMANN, G.P. Reproducibility of electromyography and ground reaction force during various running techniques. Gait and Posture, Oxford, v.19, p.115-23, 2004.

KUIPERS, H.; RIETJENS, G.; VERSTAPPEN, F.; SCHOENMARKERS, H.; HOFMAN, G. Effects of stage duration in incremental running tests on physiological variables. International Journal of Sports Medicine, Stuttgart, v.24, p. 486-91, 2003.

KYRÖLÄINEN, H.; AVELA, J.; KOMI, P.V. Changes in muscle with increasing running speed. Journal of Sports Science, London, v.23, p.1101-09, 2005.

LAVCANSKA, V.; TAYLOR, N.F.; SCHACHE, A.G. Familiarization to treadmill running in young unimpaired adults. Human Movement Science, Amsterdam, v.24, p.544-57, 2005.

MARTIN, P.E.; SANDERSON, D.J. Biomechanics of walking and running. In: GARRET, W.E.; KIRKENDAL, D. (Eds.). Exercise and sport science. Philadelphia: Lippincot Willians \& Wilkins, 2000. p.639-59.

NUMMELA, A.; RUSKO, H.; MERO, A. EMG activities and ground reaction forces during fatigued and nonfatigued sprinting. Medicine and Science in Sports and Exercise, Philadelphia, v.26, p.605-09, 1994.

SMOLIGA, J.M.; MYERS, J.B.; REDFERN, M.S.; LEPHART, S.M. Reliability and precision of EMG in leg, torso, and arm muscles during running. Journal of Electromyography and Kinesiology, Oxford, v.20, p.e1-e9, 2010.

TILLMAN, M.D.; HASS, C.J; CHOW, J.W.; BRUNT, D. Lower extremity coupling parameters during locomotion and landings. Journal of Applied Biomechanics, Champaign, v.21, p.359-70, 2005.

TSUBOI, T.; SATOU, T.; EGAWA, K.; IZUMI, Y.; MIYAZAKI, M. Spectral analysis of electromyogram in lumbar muscles: fatigue induced endurance contraction. European Journal of Applied Physiology, Berlin, v.69, p.361-66, 1994. VAN INGEN SCHENAU, G.J.; PRATT, C.A.; MacPHERSON, J.M. Differential use and control of mono- and biarticular muscles. Human Movement Science, Amsterdam, v.13, p.495-517, 1994.

VUORIMAA, T.; VIRLANDER, R.; KURKILAHTI, P.; VASANKARI, T.; HÃKKINEN, K. Acute changes in muscle activation and leg extension performance after different running exercises in elite long distance runners. European Journal of Applied Physiology, Berlin, v.96, p.282-91, 2006.

\begin{tabular}{r|l} 
ENDEREço & \\
Carina Helena Wasem Fraga & \\
Laboratório de Biomecânica & Recebido para publicação: 12/o9/2011 \\
Escola de Educação Física e Esporte - USP & Revisado: 28/05/2012 \\
Av. Prof. Melo Moraes, 65 & Aceito: 14/06/2012 \\
05508-030 - São Paulo - SP - BRASIL & \\
e-mail: wcarina_helena@hotmail.com &
\end{tabular}

610 • Rev. bras. Educ. Fís. Esporte, São Paulo, v.26, n.4, p.599-610, out./dez. 2012 